\title{
Detection and analysis of long noncoding RNA expression profiles related to epithelial- mesenchymal transition in keloids
}

\author{
Zhixiong Chen ${ }^{1}, \mathrm{Xi}_{\mathrm{Ch}} \mathrm{C}^{1,2}$ and Jinghong $\mathrm{X} \mathrm{u}^{1^{*}}$ (D)
}

\author{
*Correspondence: \\ doctorxjh@zju.edu.cn \\ ${ }^{1}$ Department of Plastic \\ Surgery, The First Affiliated \\ Hospital, Zhejiang University \\ School of Medicine, \\ Hangzhou 310003, People's \\ Republic of China \\ Full list of author information \\ is available at the end of the \\ article
}

\begin{abstract}
Background: The role of epithelial-mesenchymal transition (EMT) in the pathogenesis of keloids is currently raising increasing attention. Long noncoding RNAs (IncRNAs) govern a variety of biological processes, such as EMT, and their dysregulation is involved in many diseases including keloid disease. The aim of this study was to identify differentially expressed EMT-related IncRNAs in keloid tissues versus normal tissues and to interpret their functions.
\end{abstract}

Results: Eleven IncRNAs and 16 mRNAs associated with EMT were identified to have differential expression between keloid and normal skin tissues (fold change $>1.5$, $P<0.05)$. Gene Ontology (GO) analysis showed that these differentially expressed mRNAs functioned in the extracellular matrix, protein binding, the positive regulation of cellular processes, the Set1C/COMPASS complex and histone acetyltransferase activity. Kyoto Encyclopedia of Genes and Genomes (KEGG) analysis demonstrated that these mRNAs are involved in pathways in cancer. The IncRNA, XLOC_000587 may promote cell proliferation and migration by enhancing the expression of ENAH, while AF268386 may facilitate the invasive growth of keloids by upregulating DDR2.

Conclusions: We characterized the differential expression profiles of EMT-related IncRNAs and mRNAs in keloids, which may contribute to preventing the occurrence and development of keloids by targeting the corresponding signaling pathways. These IncRNAs and mRNAs may provide biomarkers for keloid diagnosis and serve as potential targets for the treatment of this disease.

Keywords: Keloid, Long noncoding RNA, Epithelial-mesenchymal transition

\section{Background}

Keloids are benign lesions with invasive growth beyond the original wound borders, in which the hyperproliferation of fibroblasts and excessive accumulation of extracellular matrix can be observed [1]. The claw-like extension of keloids not only causes cosmetic disfigurement, but also brings about uncomfortable feelings such as itch and pain, as well as local dysfunction, resulting in impaired emotional wellbeing and reduced quality of life. However, the exact mechanism of keloid scarring remains unknown. Any disorder during wound healing, including an enhanced inflammatory response, overexpression

(C) The Author(s) 2022. Open Access This article is licensed under a Creative Commons Attribution 4.0 International License, which permits use, sharing, adaptation, distribution and reproduction in any medium or format, as long as you give appropriate credit to the original author(s) and the source, provide a link to the Creative Commons licence, and indicate if changes were made. The images or other third party material in this article are included in the article's Creative Commons licence, unless indicated otherwise in a credit line to the material. If material is not included in the article's Creative Commons licence and your intended use is not permitted by statutory regulation or exceeds the permitted use, you will need to obtain permission directly from the copyright holder. To view a copy of this licence, visit http:// creativecommons.org/licenses/by/4.0/. The Creative Commons Public Domain Dedication waiver (http://creativecommons.org/publi cdomain/zero/1.0/) applies to the data made available in this article, unless otherwise stated in a credit line to the data. 
of growth-related factors, and excessive activation of fibroblasts, is conducive to abnormal collagen deposition in keloids [2]. Transforming growth factor- $\beta$ (TGF- $\beta$ ), connective tissue growth factor (CTGF), vascular endothelial growth factor (VEGF) and the renin-angiotensin system were reported to be potential targets in keloid management considering their participation in wound healing and keloid formation [3, 4]. In addition, miRNA modulation may be a novel therapeutic solution [5]. However, neither first-line therapies nor developing treatments could guarantee satisfying curative effects, which calls for more research on effective targets. Currently, the contribution of epithelial-mesenchymal transition (EMT) to keloid overgrowth is appealing as a potential treatment target. High expression of mesenchymal markers, such as vimentin and fibronectin, and decreased expression of the epithelial marker E-cadherin confirmed EMT phenomenon in keloids [6]. EMT refers to the biological process associated with the loss of polarity of epithelial cells and their transformation into mesenchymal cells with migratory and invasive characteristics [7]. It has been reported that inflammatory stimuli and a hypoxic microenvironment may induce the transition from keratinocytes to fibroblasts, facilitating the invasive growth of keloids [8].

Long noncoding RNAs (lncRNAs) are widely conserved sequences longer than 200 nucleotides without protein-coding capacity [9]. As an emerging class of regulatory RNAs, IncRNAs may participate in fundamental processes including cell cycle regulation, proliferation, apoptosis, self-renewal, metastasis and the DNA damage response through transcriptional or posttranscriptional regulation and thus give rise to diseases. Abnormally expressed lncRNAs play a key role in fibrosis [10] and different types of cancer, including breast cancer [11-13], lung cancer [14, 15], colorectal cancer [16, 17], esophageal squamous cell carcinoma [18], and cutaneous squamous cell carcinoma [19, 20].

The role of lncRNAs in normal skin and keloids is an emerging research topic. LncRNAs such as antidifferentiation noncoding RNA (ANCR) and terminal differentiationinduced noncoding RNA (TINCR) are critical in maintaining epidermal stability [21]. In addition to regulating normal skin formation, it has been reported that lncRNAs may influence the occurrence and development of keloids. LncRNAs are aberrantly expressed in keloid tissues compared with normal skin tissues, indicating that differentially expressed lncRNAs may participate in keloid formation [22]. Although the lncRNA expression profiles of keloids have been reported, few studies have concentrated on the physiological functions of lncRNAs. Zhu et al. [23] reported increased lncRNA-ATB expression in keloids, and revealed that lncRNA-ATB regulates the autocrine secretion of TGF- $\beta 2$ in keloid fibroblasts by downregulating ZNF217 via miR-200c. Yuan et al. [24] identified the upregulation of lncRNA LINC01116 in keloids and demonstrated that LINC01116 accelerates the development of keloids by regulating the miR-203/SMAD5 axis. In addition, differential expression profiles of lncRNAs associated with the Hedgehog and Wnt pathways suggest a possible regulatory mechanism of lncRNAs in the occurrence and development of keloids [25, 26].

Many studies have confirmed the connection between lncRNAs and EMT [27-30] in multiple diseases, especially in tumors [31-35]. Chen et al. [36] reported that lncRNA HOXA11-AS upregulation promotes EMT by inhibiting miR-200b in non-small cell lung cancer (NSCLC) and predicts a poor prognosis. LncRNA NORAD was also reported 
Table 1 Baseline data of the included patients

\begin{tabular}{llll}
\hline Sample ID & Age (years) & Sex & Lesion location \\
\hline A & 21 & Male & Cheek \\
B & 67 & Female & Abdomen \\
C & 30 & Female & Chest \\
\hline
\end{tabular}

Table 2 RNA quantification and quality assurance by NanoDrop ND-1000

\begin{tabular}{lllllcl}
\hline Sample ID & $\begin{array}{l}\text { OD260/280 } \\
\text { ratio }\end{array}$ & $\begin{array}{l}\text { OD260/230 } \\
\text { ratio }\end{array}$ & Conc. $(\mathbf{n g} / \boldsymbol{\mu l})$ & Volume $(\boldsymbol{\mu l})$ & Quantity $(\mathbf{n g})$ & $\begin{array}{l}\text { QC result } \\
\text { (pass/ } \\
\text { fail) }\end{array}$ \\
\hline A1 & 1.93 & 1.83 & 396.15 & 40 & $15,846.00$ & Pass \\
B1 & 1.93 & 1.95 & 296.42 & 30 & 8892.60 & Pass \\
C1 & 1.97 & 1.98 & 644.77 & 40 & $25,790.80$ & Pass \\
A2 & 1.90 & 2.03 & 440.80 & 40 & $17,632.00$ & Pass \\
B2 & 1.92 & 2.21 & 298.85 & 30 & 8965.50 & Pass \\
C2 & 1.92 & 1.87 & 435.73 & 40 & $17,429.20$ & Pass \\
\hline
\end{tabular}

A1-C1 refer to RNAs extracted from keloids, and A2-C2 refer to RNAs extracted from adjacent normal skin from the corresponding patients

to enhance invasion and EMT in NSCLC cells [37], while lncRNA H19 was found to facilitate the EMT and metastasis of esophageal cancer cells through the let-7c/STAT3/ EZH2/ß-catenin axis [38]. In addition, increased expression of IncRNA UCA1 plays a role in EMT occurrence by targeting miR-155 and can be used as survival prediction in cervical cancer [39]. Nevertheless, to date, no research has focused on EMT-related lncRNAs in keloids. Considering the role of EMT in the pathogenesis of keloids, this study made the first effort into detecting and analyzing EMT-related IncRNA expression profiles in keloids.

\section{Results}

\section{RNA quality testing}

The baseline data of the 3 patients included in the study are shown in Table 1. We extracted total RNA from keloids and adjacent normal skin and carried out quality testing by a NanoDrop ND-1000 spectrophotometer (Table 2). The A260/A280 ratios of RNAs extracted from the keloid and normal groups were between 1.8 and 2.1, and the A260/A230 ratios were $<1.8$, indicating good-quality RNA samples. The imaging of the agarose gel after electrophoresis demonstrated fairly sharp and intense $18 \mathrm{~s}$ and $28 \mathrm{~s}$ bands, indicating the acceptable purity and integrity of RNA samples (Fig. 1).

A1-C1 refer to RNAs extracted from keloids, and A2-C2 refer to RNAs extracted from adjacent normal skin from the corresponding patients.

\section{Differentially expressed IncRNAs and mRNAs}

A total of 773 EMT-related lncRNAs and 219 mRNAs were evaluated in the study, 11 lncRNAs and 16 mRNAs were identified to have differential expression between keloid tissue and normal skin tissue (fold change $>1.5, P<0.05$ ) (Tables 3 and 4). Among the detected lncRNAs, 7 were upregulated in keloid specimens, while 4 were downregulated. 


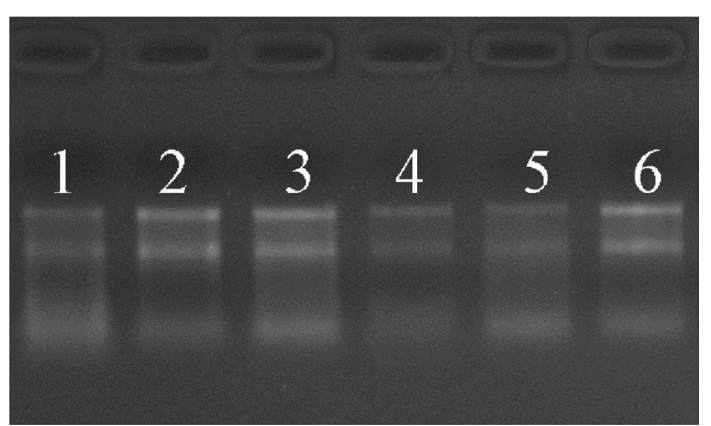

Fig. 1 RNA integrity and gDNA contamination were assessed by denaturing agarose gel electrophoresis. Lanes 1-3 correspond to RNA samples of A1-C1, and lanes 4-6 represent RNA samples of A2-C2

Table 3 Differentially expressed EMT-related IncRNAs in keloids compared with normal skin

\begin{tabular}{llcl}
\hline Seq name & Gene name & Absolute fold change & Regulation \\
\hline ENST00000539135 & RP11-264F23.3 & 2.5210468 & Up \\
uc001gch.1 & AF268386 & 4.4642715 & Up \\
AK055628 & AK055628 & 56.9629769 & Up \\
NR_033321 & MIAT & 5.7549102 & Up \\
NR_024065 & LINC00312 & 1.7928701 & Up \\
TCONS_00002241 & XLOC_000587 & 3.6222026 & Up \\
ENST00000563754 & RP11-95H3.1 & 3.3825798 & Up \\
UC003djn.3 & BC041347 & 1.6365448 & Down \\
NR_004436 & SNAR-A2 & 2.9651001 & Down \\
NR_002605 & DLEU1 & 2.1737057 & Down \\
TCONS_00014246 & XLOC_006329 & 1.7835828 & Down \\
\hline
\end{tabular}

Table 4 Differentially expressed EMT-related mRNAs in keloids compared with normal skin

\begin{tabular}{llcl}
\hline Seq name & Gene name & Absolute fold change & Regulation \\
\hline NM_001111285 & IGF1 & 2.6027441 & Up \\
NM_006475 & POSTN & 27.6007449 & Up \\
NM_013227 & ACAN & 7.4733116 & Up \\
NM_005985 & SNAI1 & 5.3111159 & Up \\
NM_003118 & SPARC & 26.7924263 & Up \\
NM_002211 & ITGB1 & 3.4373866 & Up \\
NM_003380 & VIM & 4.4300878 & Up \\
NM_019554 & S100A4 & 2.8472375 & Up \\
NM_003239 & TGFB3 & 7.59837 & Up \\
uc003pan.1 & MCM3 & 1.6558978 & Up \\
NM_004126 & GNG11 & 3.5309769 & Up \\
ENST00000304636 & COL3A1 & Up \\
NM_020061 & OPN1LW & 29.5454592 & Down \\
NM_024494 & WNT2B & 4.2499062 & Down \\
NM_017588 & WDR5 & 4.3460703 & Down \\
NM_005238 & ETS1 & 4.0202322 & Down \\
\hline
\end{tabular}


Regarding differentially expressed mRNAs, 12 were significantly upregulated, and 4 were significantly downregulated in keloids.

To compare the intensity distribution of 6 samples, we used box-whisker plots, which suggested that the data distribution from 6 chips was similar (Fig. 2). Variations in EMTrelated lncRNAs and mRNA expression among specimens are shown in volcano plots and scatter plots (Fig. 3). Based on the microarray results, hierarchical clustering was performed to infer the relationship among specimens and demonstrated distinguishable lncRNA and mRNA expression profiles between keloid tissue and normal skin tissue (Fig. 4).

By quantifying lncRNAs at the transcript level and their potential target genes at both the gene and transcript levels in parallel, microarray analysis helped to establish connections between the regulatory mechanisms and biological functions of the lncRNAs. Five pairs of predicted lncRNA-mRNA were listed as examples (Table 5). More information could be found in the additional file (see Additional file 1).

\section{Gene Ontology (GO) analysis}

Utilizing GO, the de facto standard in describing gene functions, we carried out functional annotation and enrichment analysis of differentially expressed transcripts. We found that in upregulated transcripts from keloids, the most enriched GO terms were single-organism cellular processes (biological process), the intracellular part (cellular component), and protein binding (molecular function) (Fig. 5A-C). For downregulated transcripts, the most enriched GO terms were the positive regulation of cellular processes (biological process), the Set1C/COMPASS complex (cellular component), and histone acetyltransferase activity (molecular function) (Fig. 5D-F).

\section{Kyoto Encyclopedia of Genes and Genomes (KEGG) analysis}

KEGG analysis helped us to map lncRNAs to pathways, and this analysis demonstrated a total of 10 pathways related to upregulated transcripts in keloids (Fig. 6A). The top 3 most enriched networks were hypertrophic cardiomyopathy (HCM), dilated cardiomyopathy (DCM) and pathways in cancer. Moreover, 2 pathways, corresponding to HTLV-1 infection and pathways in cancer, were found to be related to downregulated transcripts in keloids (Fig. 6B).

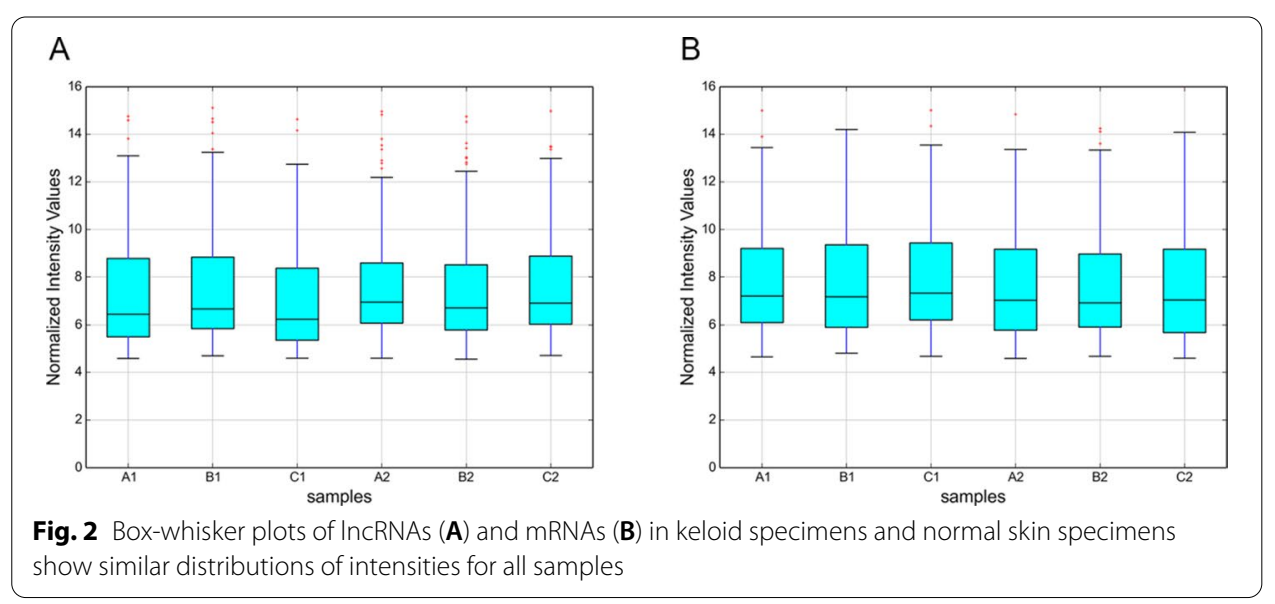




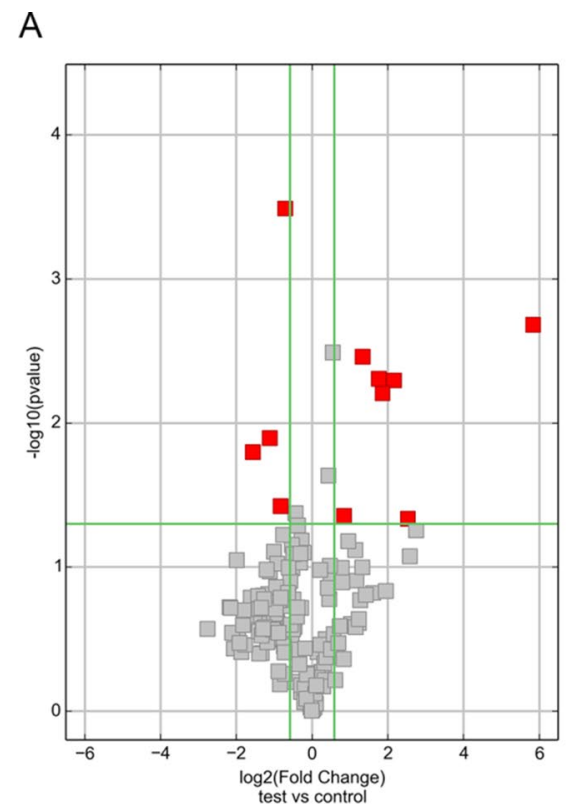

C

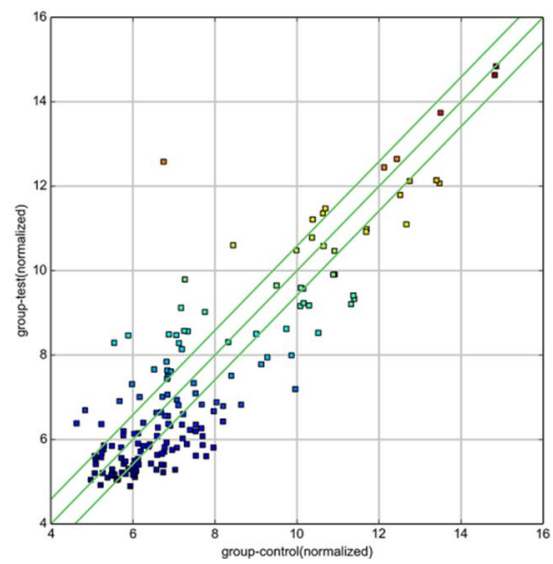

B

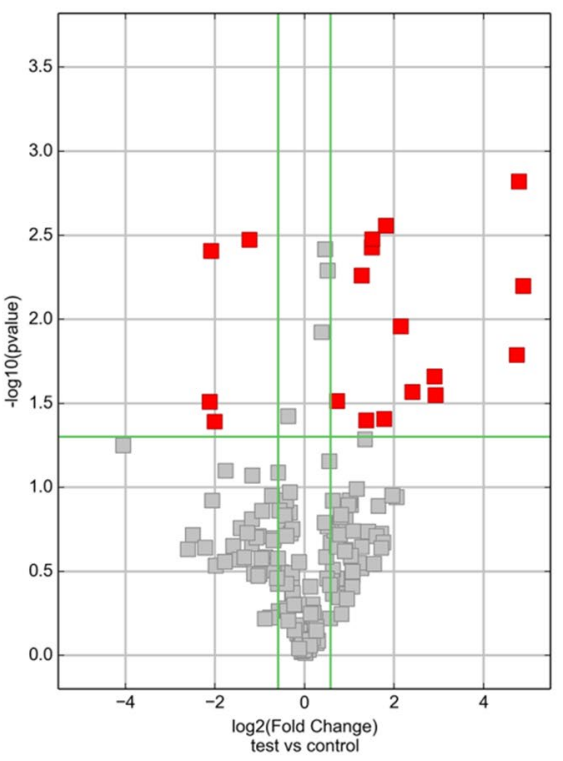

D

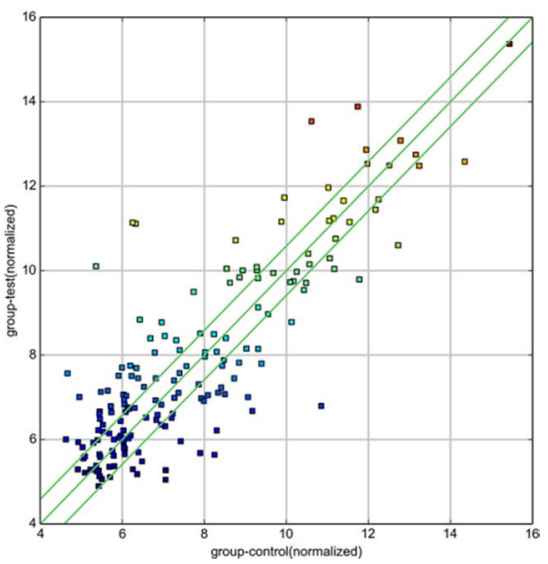

Fig. 3 Expression profiles of EMT-related IncRNAs and mRNAs in keloid specimens and normal skin specimens. Volcano plots of IncRNA (A) and mRNA (B) expression profiles showing differential expression between keloid tissue and normal skin tissue. The horizontal green line represents a $P$-value of 0.05 and the vertical green lines correspond to 1.5 -fold upregulation and downregulation. The red points represent differentially expressed RNAs with statistical significance. Scatter plots of IncRNAs (C) and mRNAs (D) are presented for the assessment of expression variation between keloid tissue and normal skin tissue. The values of the $x$ and $y$-axes are the averaged normalized signal values (log2 scaled), and the default fold change given is 1.5

\section{Validation of the microarray finding by qRT-PCR}

To validate the microarray results, we performed qRT-PCR of 5 upregulated lncRNAs (ENST00000539135, NR_033321, NR_024065, TCONS_00002241 and ENST00000563754) and 3 downregulated lncRNAs (uc003djn.3, NR_002605 and TCONS_00014246) in the original specimens (Fig. 7A). NR_033321, TCONS_00002241, ENST00000563754, and NR_002605 were proven to have significant changes between the original keloid specimens and normal skin specimens. 

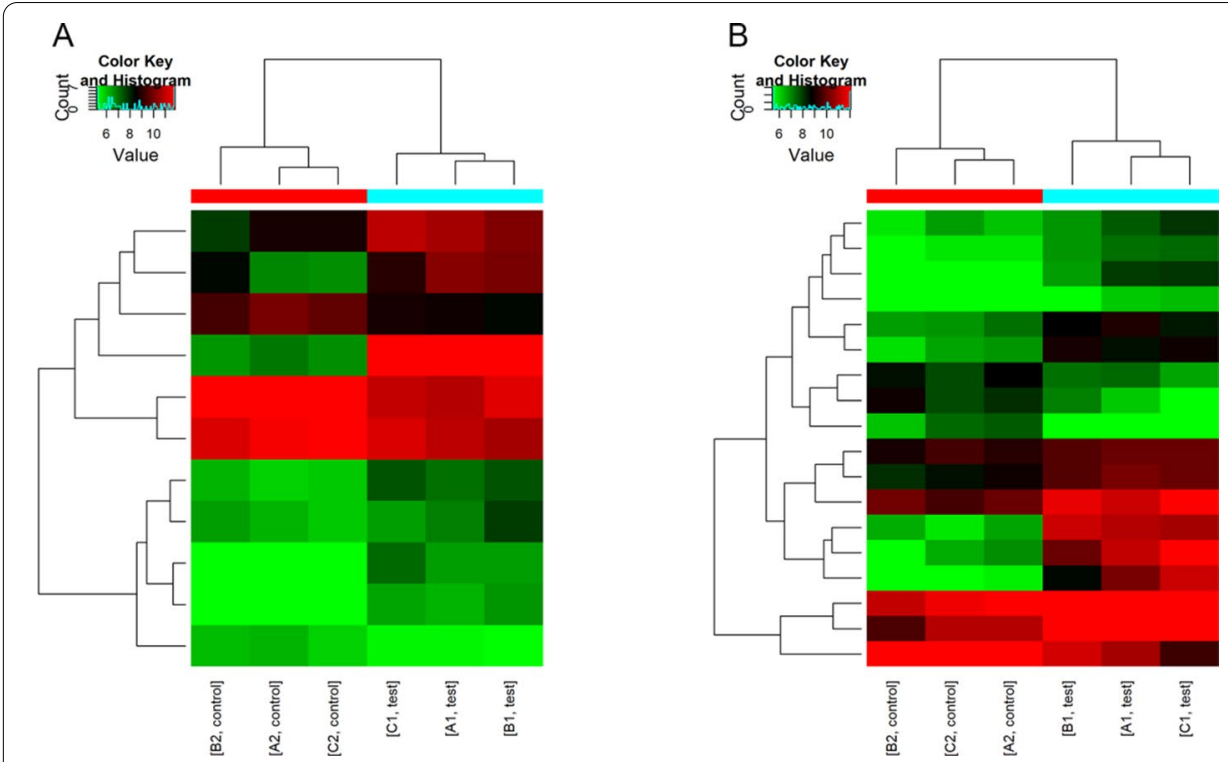

Fig. 4 Hierarchical clustering of IncRNAs (A) and mRNAs (B) classifies samples into groups according to expression level. Each column corresponds to a sample, and each row stands for a gene. Red and green colors represent high and low expression of genes, respectively

Table 5 Predicted regulation of genes by IncRNAs

\begin{tabular}{llllll}
\hline Gene symbol & Fold change & Regulation & Genomic relationship & Potential mechanism & mRNA symbol \\
\hline RP11-264F23.3 & 2.521047 & Up & Overlapping & Neighboring & CCND2 \\
AF268386 & 4.464272 & Up & Upstream & Enhancer & DDR2 \\
XLOC_000587 & 3.622203 & Up & Upstream & Enhancer & ENAH \\
MT1CP & 1.344673 & Up & Downstream & Enhancer & MT1B \\
XLOC_006329 & 1.783583 & Down & Upstream & Enhancer & PDGFA \\
\hline
\end{tabular}

Among these verified lncRNAs, TCONS_00002241 demonstrated the most apparent variation with fold change up to 112.53 .

A total of 12 pairs of keloid specimens and adjacent normal skin specimens were then tested by qRT-PCR to increase the sample size, and the expression of NR_033321, TCONS_00002241, and NR_002605 was detected (Fig. 7B). All these lncRNAs were significantly differentially expressed. Consistent with the validation results in the original specimens, TCONS_00002241 presented the most evident fold change (6.88).

\section{Discussion}

This study utilized an EMT-specific microarray and bioinformatics analysis to evaluate EMT-related lncRNAs, mRNAs, and involved signaling pathways in keloids. The results of the microarray are summarized in Tables 3 and 4 and were verified by qRT-PCR in a larger set of samples to guarantee reliability. Compared to the use of qRT-PCR alone to assess certain lncRNAs commonly investigated in cancer, microarray analysis provides a more macroscopic differential expression profile, helping to identify novel lncRNAs involved in the pathogenesis of keloid diseases. Because the functions of many lncRNAs are still elusive while corresponding mRNAs are better 


\begin{tabular}{|c|c|c|c|}
\hline$A$ & Sig GO terms of DE gene-BP & B & Sig GO terms of DE gene-CC \\
\hline Skcktat ystem decelopment [G0:0001501] & 17 enel & Extracedlualar matrix [G0:00310121 & \\
\hline Negative regulation of dec clopmental ... [G:0051093] & 17 geness & Platelet alpha gramule lumen |[C:00331093] & $\beta$ genes] \\
\hline 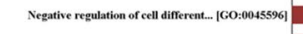 & $\square_{16 \text { geness }}$ & Phatele atpha granule [CO:0031109|] & 13 genes 1 \\
\hline 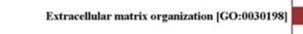 & Is genes] & 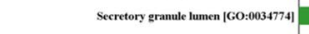 & [3 genes| \\
\hline 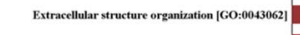 & 15 genes] & 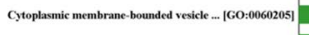 & |13 genes] \\
\hline Tisuc dectlopment [G0:00988ss] & [s genes] & Vesick lumen [ [6:0031983] ] & 13 enesel \\
\hline Hear decectopment [CO:0007507] & Is genes & 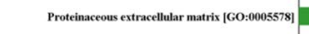 & |l genesel \\
\hline Wound healing [6:00042060)] & |s g genes & 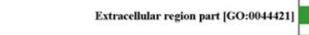 & 19 genes \\
\hline Reguhtion of cell diffrerentatition [GO:0045995] & 117 genes] & Eatracelluar space $\mid$ G0:00056 $15 \mid$ & I6 genes) \\
\hline 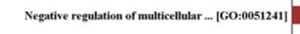 & 16 genes) & Extracectluar r region [G0:0005576] & 19 genesl \\
\hline & 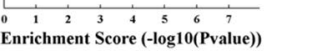 & & 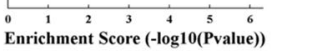 \\
\hline C & Sig GO terms of DE gene-MF & $\mathrm{D}$ & Sig GO terms of DE gene-BP \\
\hline Integrin binding [60:-0005178] & 13 genes] & 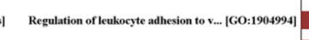 & \\
\hline Protetin complex bindinge $[60: 00324003 \mid$. & Is senes| & Iris morphogenenis [GO:00661072] & [1 genel \\
\hline 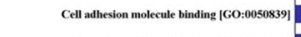 & $D_{\text {Henesel }}$ & 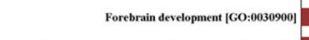 & 12 enese) \\
\hline 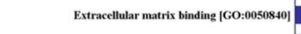 & 12 genes 1 & 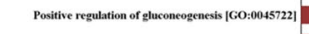 & II genel \\
\hline Collagen binding [ [G:00005sis] & ]$^{\mid 2 \text { geness } 1}$ & 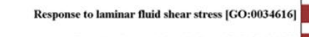 & 11 genel \\
\hline 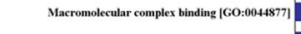 & Is genel & Protitin-chromophlore linkage [GO:001 1828s] & I 1 penel \\
\hline Extracelluhar matrix structural const.. [GO:0005201] ] & $\square_{12 \text { enesel }}$ & Reguhtiton of estracectluar matrix di.. | $60: 0010715]$ & \\
\hline CYtoline binding [ [GO:0019555| & [12 enesel & Estrous scle [60:004+1819] & 11 zenel \\
\hline Receptor binding $\mid[6: 00005102]$ & [15 genes] & Histone $\mathrm{H}+\mathrm{K}$ - ace & \\
\hline 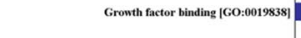 & 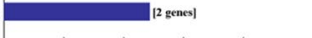 & Histone H4-K5 saceythation [G:000043981] & \\
\hline E & $\begin{array}{l}1 \\
\text { Enrichment Score (-logio(Pvalue)) } \\
\text { Sig GO terms of DE gene-CC }\end{array}$ & $\mathrm{F}$ & 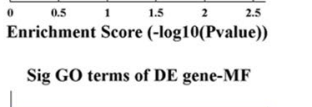 \\
\hline 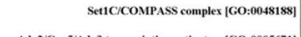 & & Hstone acetyitrans sterase activity (H... [GO:0046972] & \\
\hline 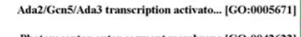 & II genel & 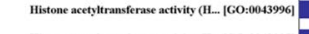 & II genel \\
\hline 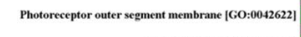 & [1 genel & 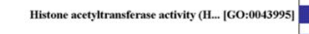 & $Z_{\text {[1 genel] }}$ \\
\hline MLL1 complex |60:0071339] & I1 enenel & 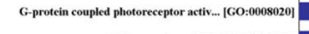 & In genel \\
\hline 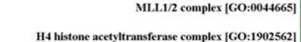 & [1 genel & Photoreceptor activity [60:00098s1] & \\
\hline 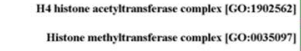 & [1 genel] & 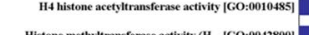 & \\
\hline 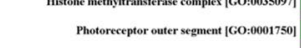 & {$\left[\begin{array}{l}\text { [1 gene] } \\
\mid 1 \text { genc| }\end{array}\right.$} & 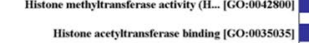 & \\
\hline CWliary membrane [G0:0060170] & [1 genel & Frizled binting [C:0:0055109] & \\
\hline Histone acestytraustersase complex [60:0000123] & [1 genel & Histone-1ysine N-methytransterase ace. [GO:0018024] & $\square_{11 \text { genel }}$ \\
\hline & 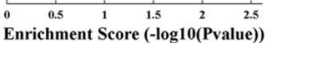 & & $\begin{array}{cccc}0.5 & 1 & 1.5 & 2 \\
0 & 2.5 \\
\text { Enrichment Score } & (-\log 10(\text { Pvalue })\end{array}$ \\
\hline \multicolumn{4}{|c|}{$\begin{array}{l}\text { Fig. } 5 \text { GO analysis of differentially expressed coding gene transcripts. The most highly enriched GO terms } \\
\text { for the upregulated transcripts in keloids in terms of biological processes }(\mathbf{A}) \text {, cellular components }(\mathbf{B}) \text {, and } \\
\text { molecular functions }(\mathbf{C}) \text {. The most highly enriched } \mathrm{GO} \text { terms for the downregulated transcripts in terms of } \\
\text { biological processes }(\mathbf{D}) \text {, cellular components }(\mathbf{E}) \text {, and molecular functions }(\mathbf{F})\end{array}$} \\
\hline
\end{tabular}

A

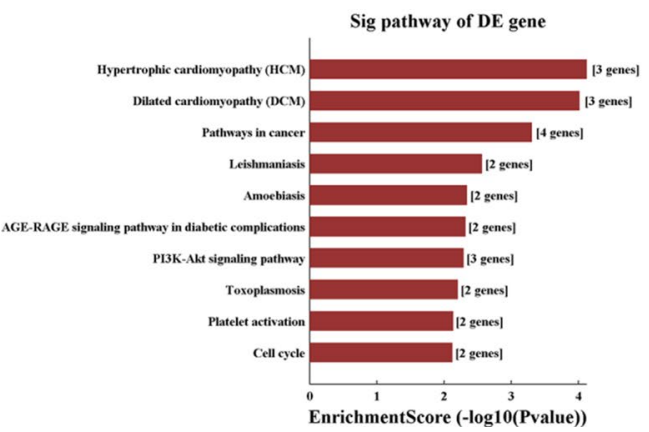

B

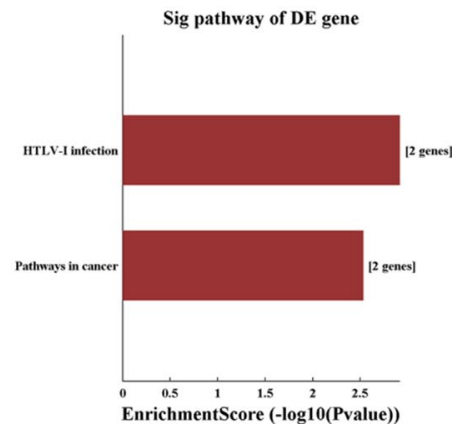

Fig. 6 Pathway analysis. Pathways related to upregulated (A) and downregulated (B) transcripts in keloids 

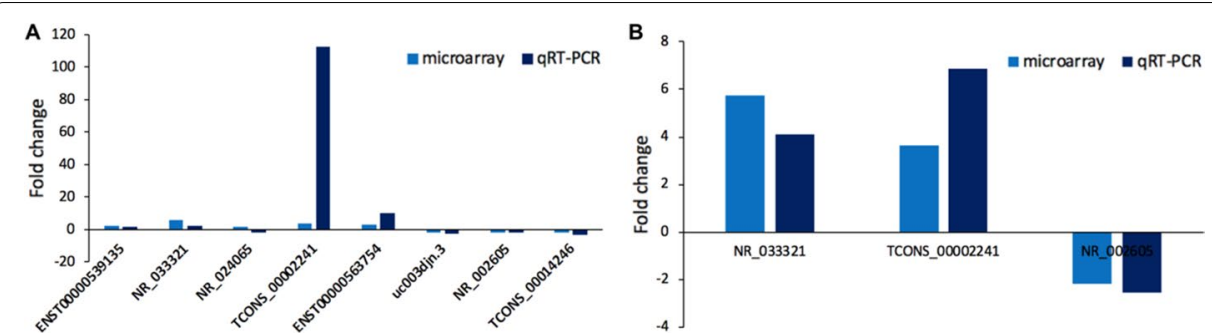

Fig. 7 Relative expression of candidate IncRNAs detected by qRT-PCR. A Comparison of the fold changes of 8 IncRNAs between microarray and qRT-PCR results in original specimens. B Validation of 3 chosen IncRNAs by qRT-PCR in an enlarged sample set

understood, we conducted GO and KEGG analyses of target gene mRNAs to analyze the regulatory mechanism and biological function of lncRNAs in keloids. GO analysis showed that the functions of these mRNAs were related to the extracellular matrix, protein binding, the positive regulation of cellular process, the Set1C/COMPASS complex, and histone acetyltransferase activity, suggesting that the differentially expressed lncRNAs and mRNAs play a role in the development of tumors. Following GO analysis, the subsequent KEGG analysis demonstrated that the mRNAs are involved in pathways in cancer. Considering that keloids are benign lesions characterized by invasive growth, it is reasonable that pathways in cancer are recognized to be differentially enriched between keloids and normal skin tissue.

Notably, XLOC_000587, the corresponding gene of TCONS_00002241, is located within $300 \mathrm{~kb}$ upstream of ENAH and may be an enhancer of ENAH. ENAH is an actin regulatory protein involved in cell motility and adhesion. Extensive studies have demonstrated upregulation of ENAH in many tumors, including breast carcinoma [40], cervical carcinoma [41], colorectal carcinoma [42], and hepatocellular carcinoma [43]. Notably, the expression of ENAH is correlated with vascular invasion, tumor grade, and unfavorable prognosis, which suggests that ENAH might participate in carcinogenesis and tumor progression [44-46]. Chen et al. [45] reported that EMT may be involved in the role of ENAH in gastric cancer cell proliferation and metastasis. EMT is regulated by a complex network including the TGF- $\beta$, Wnt, and Notch signaling pathways [47], while ENAH has been demonstrated to function as a transcriptional target of the Wnt/ $\beta$-catenin pathway and a novel connection between the Wnt/ $\beta$-catenin and Notch signaling cascades [48]. Therefore, we speculate that XLOC_000587 in keloids may further regulate related signaling pathways by increasing the expression of ENAH to promote EMT and the invasive development of keloids. Elucidating the mechanism by which XLOC_000587 participates in EMT is expected to provide new insights into keloid treatment.

In addition, AF268386 was predicted to be an enhancer of discoidin domain receptor 2 (DDR2), a tyrosine kinase receptor activated by collagen [49]. As a recognized critical regulator of EMT, DDR2 upregulation has been confirmed in many tumor cell lines and takes part in the interaction with the extracellular matrix, the regulation of cell motility and adhesion, and metastatic spreading [49-52]. Toy et al. [53] reported that overexpressed DDR2 in triple-negative breast cancer may indicate carcinoma 
with worse prognosis. Moreover, Ren et al. [54] reported that DDR2 expression was closely correlated with the expression of the hypoxic marker HIF-1 $\alpha$ in breast cancer specimens, and hypoxia treatment could induce the expression of DDR2 in human breast cancer cell lines. Regarding wound healing, DDR2 is capable of promoting proliferation, collagen I secretion and MMP2 production in skin fibroblasts, and a delay in wound healing was observed in DDR2 null mice [55]. According to the role of DDR2 in wound healing and metastatic spreading and the hypoxic microenvironment in keloids, we hypothesize that targeting AF268386 or the corresponding lncRNA AF268386 could be a potential therapeutic approach for keloids treatment. In view of the limited research findings, more studies concerning these candidate lncRNAs remain to be carried out to uncover underlying pathways, establish lncRNAmiRNA-mRNA molecular network and clarify the exact functions in the occurrence and development of keloids.

\section{Conclusion}

This study identified and verified the differential expression of EMT-related lncRNAs in keloids, indicating that lncRNAs are likely to be involved in EMT and the overgrowth of keloids. In particular, lncRNA XLOC_000587 may participate in cell proliferation and migration by enhancing the expression of ENAH, and AF268386 may accelerate the invasive development of keloids by upregulating DDR2. The differentially expressed lncRNAs will hopefully provide novel insights into the pathogenesis of keloid diseases by multiple mechanisms such as signal transduction and competing endogenous RNA (ceRNA) networks.

\section{Methods}

\section{Patients and specimens}

The study complied with the Declaration of Helsinki of the World Medical Association and was approved by the Ethics Committee of The First Affiliated Hospital, Zhejiang University School of Medicine. All 12 patients were fully informed of the aim and protocol of the study, gave written informed consent, and provided excised keloids and adjacent normal skin for scientific research and publication. None of the patients had previously undergone radiotherapy or drug therapy. The keloids had clinical characteristics of overgrowth beyond the original wound boundaries and were confirmed by pathology. Tissues were frozen in liquid nitrogen and stored at $-80^{\circ} \mathrm{C}$ immediately after excision.

\section{RNA extraction and quality control}

Total RNA was extracted from frozen specimens using TRIzol reagent (Invitrogen, Carlsbad, CA, USA) following the manufacturer's instructions (Invitrogen). The RNA quality was assessed by NanoDrop ND-1000 (Thermo Fisher Scientific, Waltham, MA, USA), and RNA integrity was assessed by denaturing agarose gel electrophoresis.

\section{RNA labeling and array hybridization}

Keloid and normal skin specimens from 3 randomly selected patients were tested by microarray profiling. The chip used was the lncPath Human EMT Array $(8 \times 15 \mathrm{~K})$ 
(ArrayStar, Rockville, MD, USA). Microarray analysis was performed by Kangchen Biology Engineering Co., Ltd., (Shanghai, China) according to the manufacturer's protocol (Arraystar). LncRNAs/mRNAs were purified from total RNA after removal of rRNA (Arraystar rRNA removal kit). Then, each sample was amplified and transcribed into fluorescent cRNA along the entire length of the transcripts without $3^{\prime}$ bias utilizing a random priming method (Arraystar Flash RNA Labeling Kit, Arraystar). The labeled cRNAs were purified by RNeasy Mini Kit (Qiagen, Germany). The concentration and

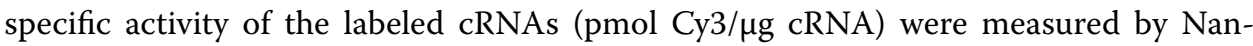
oDrop ND-1000. One microgram of each labeled cRNA was fragmented by adding $5 \mu \mathrm{l}$ $10 \times$ Blocking Agent and $1 \mu \mathrm{l} 25 \times$ Fragmentation Buffer and then heating the mixture at $60{ }^{\circ} \mathrm{C}$ for $30 \mathrm{~min}$. Finally, $25 \mu \mathrm{l} 2 \times \mathrm{GE}$ Hybridization buffer was added to dilute the labeled cRNA. Fifty microliters of hybridization solution was dispensed into the gasket slide and assembled into the lncRNA expression microarray slide. The slides were incubated for $17 \mathrm{~h}$ at $65{ }^{\circ} \mathrm{C}$ in an Agilent Hybridization Oven. The hybridized arrays were washed, fixed and scanned using the Agilent Scanner G2505C.

\section{Data analysis}

Differentially expressed lncRNAs/mRNAs were defined as those with a fold change $\geq 1.5$ (upregulated or downregulated) combined with $P$-value $<0.05$. Agilent Feature Extraction software (version 11.0.1.1) was used to analyze acquired array images. Quantile normalization and subsequent data processing were performed using the $\mathrm{R}$ software limma package. Differentially expressed lncRNAs/mRNAs with statistical significance between two groups were identified through volcano plot filtering. Differentially expressed lncRNAs/mRNAs between two samples were identified through fold change filtering. Hierarchical clustering was performed to show the distinguishable lncRNA/mRNA expression pattern among samples.

Based on the Gene Ontology (GO) database (http://www.geneontology.org), the differential transcripts underwent enrichment analysis of gene function via $\mathrm{GO}$ analysis, which indicates enriched terms in the categories of biological process, molecular function and cellular component. Pathway analysis of identified differentially expressed genes was performed using the Kyoto Encyclopedia of Genes and Genomes (KEGG) database (http://www.genome.jp/kegg/).

\section{Real-time quantitative polymerase chain reaction (qRT-PCR)}

Reverse transcription of extracted RNA was performed utilizing SuperScript ${ }^{\mathrm{TM}}$ III reverse transcriptase (Invitrogen) following the manufacturer's instructions. qRT-PCR was conducted using the ethidium bromide staining method with ViiA 7 real-time PCR system (Applied Biosystems) following the manufacturer's protocols.

Five significantly upregulated (ENST00000539135, NR_033321, NR_024065, TCONS_00002241 and ENST00000563754) and 3 downregulated lncRNAs (uc003djn.3, NR_002605 and TCONS_00014246) were selected for qRT-PCR validation in the original specimens. NR_033321, NR_002605 and TCONS_00002241 were further detected in a larger set of samples including 12 pairs of keloid tissues and adjacent normal skin tissues. $\beta$-Actin was used as endogenous control. The primer sequences were as follows:

$\beta$-actin: 
Forward: 5' GTGGCCGAGGACTTTGATTG3'

Reverse: 5' CCTGTAACAACGCATCTCATATT3'

ENST00000539135:

Forward: 5' AGCCAAGAAACGGTCCAGA 3'

Reverse: $5^{\prime}$ AGCACAGGTAGAGATCAGGAGG $3^{\prime}$

NR_033321:

Forward: 5' GACCCGAGTTGGAGGCATCT 3'

Reverse: $5^{\prime}$ TGTGGTTGTGAACGTCCTGAAG3'

NR_024065:

Forward: 5' GCTATTAGAGGGTGTTGCTGAC3'

Reverse: $5^{\prime}$ TGTATTGTGGCTATGTGGGAG $3^{\prime}$

TCONS_00002241:

Forward: 5' CACATTTACAGACTACAGAGCC $3^{\prime}$

Reverse: 5' CTGACCACACCATCAGCAAC 3'

ENST00000563754:

Forward: 5' CATTCCTGAGGCTGTTCGT $3^{\prime}$

Reverse: 5' GAAAATGTCTCTGAGAACCCAT $3^{\prime}$

uc003djn.3:

Forward: 5' AGCACAGCCTGAAACCCAA $3^{\prime}$

Reverse: 5' AGGAAGCATCTCACCCTCCT $3^{\prime}$

NR_002605:

Forward: 5' CCTTGTATATGGATACACGTGC $3^{\prime}$

Reverse: $5^{\prime}$ TCCTTTCTGGTAGAATCACTGG $3^{\prime}$

TCONS_00014246:

Forward: 5' CCGAAAAGCGAACAGTCCA $3^{\prime}$

Reverse: 5' CGTGTTTGGTGAGTTCGGTT $3^{\prime}$.

The reaction conditions for PCR were as follows: $95^{\circ} \mathrm{C}$ for $10 \mathrm{~min}$, then 40 PCR cycles at $95{ }^{\circ} \mathrm{C}$ for $10 \mathrm{~s}$ and $60^{\circ} \mathrm{C}$ for $60 \mathrm{~s}$. Products were slowly heated from 60 to $99^{\circ} \mathrm{C}$ after amplification, and melting curve was established to test the specificity of PCR products. The corrected relative expression level was acquired by the following calculation, the concentration of the target gene divided by the concentration of the housekeeping gene.

\section{Statistical analysis}

All data were analyzed utilizing SPSS (version 18.0; SPSS, Inc., Chicago, IL, USA). The Student's $t$-test was performed to evaluate the differences between distinct groups, and one-way ANOVA and SNK methods were used for comparisons among groups. A $P$-value $<0.05$ was considered significant.

\section{Abbreviations}

EMT: Epithelial-mesenchymal transition; IncRNA: Long noncoding RNA; qRT-PCR: Real-time quantitative polymerase chain reaction; GO: Gene Ontology; KEGG: Kyoto Encyclopedia of Genes and Genomes; TGF- $\beta$ :Transforming growth factor- $\beta$; CTGF: Connective tissue growth factor; VEGF: Vascular endothelial growth factor; ANCR: Antidifferentiation noncoding RNA; TINCR: Terminal differentiation-induced noncoding RNA; NSCLC: Non-small cell lung cancer; HCM: Hypertrophic cardiomyopathy; DCM: Dilated cardiomyopathy; DDR2: Discoidin domain receptor 2. 


\section{Supplementary Information}

The online version contains supplementary material available at https://doi.org/10.1186/s12938-022-00976-x.

Additional file 1. Predicted regulation of 100 pairs of IncRNA-mRNA.

\section{Acknowledgements}

None.

\section{Authors' contributions}

ZXC performed the experimental research and data analysis, and wrote and edited the manuscript. XC performed the experimental research and data analysis. JHX supervised the research and reviewed and edited the manuscript. All authors read and approved the final manuscript.

\section{Funding}

This work was supported by Zhejiang Provincial Natural Science Foundation of China under Grant No. Q21H150018 and Zhejiang Provincial Postdoctoral Project.

Availability of data and materials

The microarray datasets in the present study are available on GEO with accession number GSE182192.

\section{Declarations}

\section{Ethical approval and consent to participate}

The study complied with the Declaration of Helsinki of the World Medical Association, and was approved by the Ethics Committee of The First Affiliated Hospital, Zhejiang University School of Medicine. All 12 patients were fully informed of the aim and protocol of the study, and gave written informed consent to provide excised keloid tissues and adjacent normal skin tissues for scientific research and publication. All institutional and national guidelines for the care and use of laboratory animals were followed.

\section{Consent for publication}

Not applicable.

\section{Competing interests}

The authors declare no conflict of interest.

\section{Author details}

${ }^{1}$ Department of Plastic Surgery, The First Affiliated Hospital, Zhejiang University School of Medicine, Hangzhou 310003, People's Republic of China. ${ }^{2}$ Zhejiang University School of Medicine, Hangzhou 310000, People's Republic of China.

Received: 26 August 2021 Accepted: 3 January 2022

Published online: 11 January 2022

\section{References}

1. Jagdeo J, Kerby E, Glass DA II. Keloids are large, firm, raised scars that occur after skin injury. JAMA Dermatol. 2021;157(6):744

2. Berman B, Maderal A, Raphael B. Keloids and hypertrophic scars: pathophysiology, classification, and treatment. Dermatol Surg. 2017:43:S3-18.

3. Naik PP. Review on novel targets and therapies for Keloids. Clin Exp Dermatol. 2021. https://doi.org/10.1111/ced. 14920.

4. Hedayatyanfard K, Haddadi N-S, Ziai SA, Karim H, Niazi F, Steckelings UM, et al. The renin-angiotensin system in cutaneous hypertrophic scar and keloid formation. Exp Dermatol. 2020;29(9):902-9.

5. Stevenson AW, Deng Z, Allahham A, Prele CM, Wood FM, Fear MW. The epigenetics of keloids. Exp Dermatol. 2021;30(8):1099-114.

6. Ma XY, Chen J, Xu B, Long X, Qin H, Zhao RC, et al. Keloid-derived keratinocytes acquire a fibroblast-like appearance and an enhanced invasive capacity in a hypoxic microenvironment in vitro. Int J Mol Med. 2015;35(5):1246-56.

7. Yuan FL, Sun ZL, Feng Y, Liu SY, Du Y, Yu S, et al. Epithelial-mesenchymal transition in the formation of hypertrophic scars and keloids. J Cell Physiol. 2019;234(12):21662-9.

8. Kuwahara H, Tosa M, Egawa S, Murakami M, Mohammad G, Ogawa R. Examination of epithelial mesenchymal transition in keloid tissues and possibility of keloid therapy target. Plast Reconstr Surg Glob Open. 2016. https://doi.org/ 10.1097/GOX.0000000000001138.

9. Borkiewicz L, Kalafut J, Dudziak K, Przybyszewska-Podstawka A, Telejko I. Decoding LncRNAs. Cancers. 2021. https:// doi.org/10.3390/cancers13112643.

10. Xu Q, Cheng D, Liu Y, Pan H, Li G, Li P, et al. LncRNA-ATB regulates epithelial-mesenchymal transition progression in pulmonary fibrosis via sponging miR-29b-2-5p and miR-34c-3p. J Cell Mol Med. 2021;25(15):7294-306.

11. Li Z, Qian Z, Chen F, Jiang S, Meng L, Chen J. Identification of key IncRNA-mRNA pairs and functional IncRNAs in breast cancer by integrative analysis of TCGA data. Front Genet. 2021. https://doi.org/10.3389/fgene.2021.709514.

12. Fang $K$, Xu Z-J, Jiang S-X, Tang D-S, Yan C-S, Deng Y-Y, et al. IncRNA FGD5-AS1 promotes breast cancer progression by regulating the hsa-miR-195-5p/NUAK2 axis. Mol Med Rep. 2021. https://doi.org/10.3892/mmr.2021.12099. 
13. Liu J, Zhao G, Liu X-L, Zhang G, Zhao S-Q, Zhang S-L, et al. Progress of non-coding RNAs in triple-negative breast cancer. Life Sci. 2021. https://doi.org/10.1016/j.lfs.2021.119238.

14. Wan Y, Yao D, Fang F, Wang Y, Wu G, Qian Y. LncRNA WT1-AS downregulates IncRNA UCA1 to suppress non-small cell lung cancer and predicts poor survival. BMC Cancer. 2021. https://doi.org/10.1186/s12885-020-07767-4.

15. Chen Y, Zhou X, Huang C, Li L, Qin Y, Tian Z, et al. LncRNA PART1 promotes cell proliferation and progression in nonsmall-cell lung cancer cells via sponging miR-17-5p. J Cell Biochem. 2021;122(3-4):315-25.

16. Wu Y, Cong L, Chen W, Wang X, Qiu F. IncRNA LINC00963 downregulation regulates colorectal cancer tumorigenesis and progression via the miR-10b/FGF13 axis. Mol Med Rep. 2021. https://doi.org/10.3892/mmr.2021.11850.

17. Wang M, Zhang Z, Pan D, Xin Z, Bu F, Zhang Y, et al. Circulating IncRNA UCA1 and IncRNA PGM5-AS1 act as potential diagnostic biomarkers for early-stage colorectal cancer. 2021. Biosci Rep. https://doi.org/10.1042/BSR20211115.

18. Pan Q, Li B, Zhang J, Du X, Gu D. LncRNA THAP9-AS1 accelerates cell growth of esophageal squamous cell carcinoma through sponging miR-335-5p to regulate SGMS2. Pathol Res Pract. 2021. https://doi.org/10.1016/j.prp.2021. 153526.

19. Lu D, Sun L, Li Z, Mu Z. InCRNA EZR-AS1 knockdown represses proliferation, migration and invasion of CSCC via the PI3K/AKT signaling pathway. Mol Med Rep. 2021. https://doi.org/10.3892/mmr.2020.11714.

20. Xu Y, Dong Y, Deng Y, Qi Q, Wu M, Liang H, et al. Identifying an IncRNA-related ceRNA network to reveal novel targets for a cutaneous squamous cell carcinoma. Biology. 2021. https://doi.org/10.3390/biology10050432.

21. Hombach S, Kretz M. The non-coding skin: exploring the roles of long non-coding RNAs in epidermal homeostasis and disease. BioEssays. 2013;35(12):1093-100.

22. Liang $X$, Ma L, Long $X$, Wang X. LncRNA expression profiles and validation in keloid and normal skin tissue. Int J Oncol. 2015;47(5):1829-38.

23. Zhu H-Y, Bai W-D, Li C, Zheng Z, Guan H, Liu J-Q, et al. Knockdown of IncRNA-ATB suppresses autocrine secretion of TGF-beta 2 by targeting ZNF217 via miR-200c in keloid fibroblasts. Sci Rep. 2016. https://doi.org/10.1038/srep24728.

24. Yuan W, Sun H, Yu L. Long non-coding RNA LINC01116 accelerates the progression of keloid formation by regulating miR-203/SMAD5 axis. Burns. 2021;47(3):665-75.

25. Huang H, Fu S, Liu D. Detection and analysis of the hedgehog signaling pathway-related long non-coding RNA (IncRNAs) expression profiles in keloid. Med Sci Monit. 2018;24:9032-44.

26. Sun X-j, Wang Q, Guo B, Liu X-y, Wang B. Identification of skin-related IncRNAs as potential biomarkers that involved in Wnt pathways in keloids. Oncotarget. 2017;8(21):34236-44.

27. Zhou J, Jiang H. Livin is involved in TGF-beta 1-induced renal tubular epithelial-mesenchymal transition through IncRNA-ATB. Ann Transl Med. 2019. https://doi.org/10.21037/atm.2019.08.29.

28. Peng P-H, Lai JC-Y, Hsu K-W, Wu K-J. Hypoxia-induced IncRNA RP11-390F43 promotes epithelial-mesenchymal transition (EMT) and metastasis through upregulating EMT regulators. Cancer Lett. 2020:483:35-45.

29. Liu Y, Li Y, Xu Q, Yao W, Wu Q, Yuan J, et al. Long non-coding RNA-ATB promotes EMT during silica-induced pulmonary fibrosis by competitively binding miR-200c. Biochim Biophys Acta Mol Basis Dis. 2018;1864(2):420-31.

30. Fan Y, Zhao X, Ma J, Yang L. LncRNA GAS5 competitively combined with miR-21 regulates PTEN and influences EMT of peritoneal mesothelial cells via Wnt/beta-catenin signaling pathway. Front Physiol. 2021;12:654951.

31. Chen P-P, Zhang Z-S, Wu J-C, Zheng J-F, Lin F. LncRNA SNHG12 promotes proliferation and epithelial mesenchymal transition in hepatocellular carcinoma through targeting HEG1 via miR-516a-5p. Cell Signal. 2021. https://doi.org/10 1016/j.cellsig.2021.109992.

32. Expression of concern: long non coding RNA FAM3D-AS1 inhibits development of colorectal cancer through NF-kB signaling pathway. Biosci Rep. 2021;41(4)

33. He L, Yang H, Zhu X-L, Zhang Y, Lv K. Knockdown of long non-coding RNA SLC8A1-AS1 attenuates cell invasion and migration in glioma via suppression of Wnt/beta-catenin signaling pathways. Brain Res Bull. 2021;176:112-20.

34. Su Y, Zhou L, Yu Q, Lu J, Liu W. Long non-coding RNA LOC648987 promotes proliferation and metastasis of renal cell carcinoma by regulating epithelial-mesenchymal transition. Technol Cancer Res Treat. 2021. https://doi.org/10.1177/ 1533033821997834

35. Sun Y, Gao X, Li P, Song L, Shi L. LncRNA ZFAS1, as a poor prognostic indicator, promotes cell proliferation and epithelial-mesenchymal transition in endometrial carcinoma. Pers Med. 2021;18(1):43-53.

36. Chen JH, Zhou LY, Xu S, Zheng YL, Wan YF, Hu CP. Overexpression of IncRNA HOXA11-AS promotes cell epithelialmesenchymal transition by repressing miR-200b in non-small cell lung cancer. Cancer Cell Int. 2017. https://doi.org/ 10.1186/s12935-017-0433-7.

37. Geng QQ, Li ZB, Li XT, Wu YH, Chen NZ. LncRNA NORAD, sponging miR-363-3p, promotes invasion and EMT by upregulating PEAK1 and activating the ERK signaling pathway in NSCLC cells. J Bioenerg Biomembr. 2021. https:// doi.org/10.1007/s10863-021-09892-6.

38. Chen M-J, Deng J, Chen C, Hu W, Yuan Y-C, Xia Z-K. LncRNA H19 promotes epithelial mesenchymal transition and metastasis of esophageal cancer via STAT3/EZH2 axis. Int J Biochem Cell Biol. 2019:113:27-36.

39. Yang TJ, Wang L, Zhang Y, Zheng JD, Liu L. LncRNA UCA1 regulates cervical cancer surviva and EMT occurrence by targeting miR-155. Eur Rev Med Pharmacol Sci. 2020;24(19):9869-79.

40. Di Modugno F, Mottolese M, Di Benedetto A, Conidi A, Novelli F, Perracchio L, et al. The cytoskeleton regulatory protein hMena (ENAH) is overexpressed in human benign breast lesions with high risk of transformation and human epidermal growth factor receptor-2-positive/hormonal receptor-negative tumors. Clin Cancer Res. 2006;12(5):1470-8.

41. Gurzu S, Jung I, Prantner I, Chira L, Ember I. The immunohistochemical aspects of protein Mena in cervical lesions. Rom J Morphol Embryol. 2009;50(2):213-6.

42. Toyoda A, Kawana H, Azuhata K, Yu J, Omata A, Kishi H, et al. Aberrant expression of human ortholog of mammalian enabled (hMena) in human colorectal carcinomas: Implications for its role in tumor progression. Int J Oncol. 2009:34(1):53-60.

43. Hu K, Wang J, Yao Z, Liu B, Lin Y, Liu L, et al. Expression of cytoskeleton regulatory protein Mena in human hepatocellular carcinoma and its prognostic significance. Med Oncol. 2014. https://doi.org/10.1007/s12032-014-0939-y. 
44. Gurzu S, Krause M, Ember I, Azamfirei L, Gobel G, Feher K, et al. Mena, a new available marker in tumors of salivary glands? Eur J Histochem. 2012;56(1):39-44.

45. Chen $D, X u L, L i X, C h u Y$, Jiang M, Xu B, et al. Enah overexpression is correlated with poor survival and aggressive phenotype in gastric cancer. Cell Death Dis. 2018. https://doi.org/10.1038/s41419-018-1031-X.

46. Wang D-D, Jin Q, Wang L-L, Han S-F, Chen Y-B, Sun G-D, et al. The significance of ENAH in carcinogenesis and prognosis in gastric cancer. Oncotarget. 2017:8(42):72466-79.

47. Yang J, Weinberg RA. Epithelial-mesenchymal transition: at the crossroads of development and tumor metastasis. Dev Cell. 2008;14(6):818-29.

48. Najafov A, Seker T, Even I, Hoxhaj G, Selvi O, Ozel DE, et al. MENA is a transcriptional target of the Wnt/beta-catenin pathway. PLoS ONE. 2012. https://doi.org/10.1371/journal.pone.0037013.

49. Leitinger B. Molecular analysis of collagen binding by the human discoidin domain receptors, DDR1 and DDR2identification of collagen binding sites in DDR2. J Biol Chem. 2003;278(19):16761-9.

50. Badiola I, Villace P, Basaldua I, Olaso E. Downregulation of discoidin domain receptor 2 in A375 human melanoma cells reduces its experimental liver metastasis ability. Oncol Rep. 2011;26(4):971-8.

51. Walsh LA, Nawshad A, Medici D. Discoidin domain receptor 2 is a critical regulator of epithelial-mesenchymal transition. Matrix Biol. 2011;30(4):243-7.

52. Wang $Y-G, X u L$, Jia R-R, Wu Q, Wang T, Wei J, et al. DDR2 induces gastric cancer cell activities via activating mTORC2 signaling and is associated with clinicopathological characteristics of gastric cancer. Dig Dis Sci. 2016;61 (8):2272-83.

53. Toy KA, Valiathan RR, Nunez F, Kidwell KM, Gonzalez ME, Fridman R, et al. Tyrosine kinase discoidin domain receptors DDR1 and DDR2 are coordinately deregulated in triple-negative breast cancer. Breast Cancer Res Treat. 2015;150(1):9-18.

54. Ren T, Zhang W, Liu X, Zhao H, Zhang J, Zhang J, et al. Discoidin domain receptor 2 (DDR2) promotes breast cancer cell metastasis and the mechanism implicates epithelial-mesenchymal transition programme under hypoxia. J Pathol. 2014;234(4):526-37.

55. Olaso E, Labrador JP, Wang LH, Ikeda K, Eng FJ, Klein R, et al. Discoidin domain receptor 2 regulates fibroblast proliferation and migration through the extracellular matrix in association with transcriptional activation of matrix metalloproteinase-2. J Biol Chem. 2002;277(5):3606-13.

\section{Publisher's Note}

Springer Nature remains neutral with regard to jurisdictional claims in published maps and institutional affiliations.

- fast, convenient online submission

- thorough peer review by experienced researchers in your field

- rapid publication on acceptance

- support for research data, including large and complex data types

- gold Open Access which fosters wider collaboration and increased citations

- maximum visibility for your research: over 100M website views per year

At BMC, research is always in progress.

Learn more biomedcentral.com/submissions 